\title{
FUNÇÃO GAMA GENERALIZADA PARA DESCREVER A DISTRIBUIÇÃO DIAMÉTRICA DE UM POVOAMENTO DE EUCALIPTO
}

\author{
Renato Vinícius Oliveira Castro ${ }^{1}$, Carlos Alberto Araújo Júnior ${ }^{2}$, Hélio Garcia Leite ${ }^{3}$, \\ Ana Flávia Neves Mendes Castro ${ }^{4}$, Gilciano Saraiva Nogueira ${ }^{5}$, Lid io mar Soares Costa ${ }^{6} *$ \\ ${ }^{1}$ Universidade Federal de São João del Rey, Departamento de Ciências Agrárias, Campus Sete Lagoas, Sete Lagoas, Minas Gerais, Brasil - \\ castrorvo@ymail.com, mendesafn@hotmail.com \\ ${ }^{2}$ Universidade Federal de Viçosa, Programa de Pós-Graduação em Ciência Florestal, Viçosa, Minas Gerais, Brasil - araujocaj@ gmail.com \\ ${ }^{3}$ Universidade Federal de Viçosa, Departamento de Engenharia Florestal, Viçosa, Minas Gerais, Brasil - hgleite@ufv.br \\ ${ }^{4}$ Universidade Federal dos Vales do Jequitinhonha e Mucuri, Depart amento de Engenharia Florestal, Campus JK, Diamantina, Minas Gerais, \\ Brasil - nogueirags@gmail.com \\ ${ }^{5}$ Universidade de Brasília, Ciência Florestal, Programa de Pós-Graduação em Ciência Florestal, Campus Universitário Darcy Ribeiro, \\ Brasília, Distrito Federal, Brasil - lidiomar.ef@gmail.com*
}

Recebido para publicação: 27/03/2015 - Aceito para publicação: 06/11/2015

\begin{abstract}
Resumo
Este trabalho centra-se na estimativa de parâmetros da funcão Gama Generalizada para descrever a distribuicão de diâmetros de um povoamento equiâneo de eucalipto não desbastado e avaliar a realidade bioló gica de um sistema de equações para projetar tais parâmetros e descrever essa distribuição em uma idade futura. Foram utilizadas 48 parcelas com quatro medições anuais, provenientes de um experimento instalado em um povoamento do híbrido Eucalyptus urophylla x Eucalyptus grandis, localizados na região nordeste do estado da Bahia. O sistema de eauacões vara proiecão dos parâmetros foi avaliado através dos coeficientes de correlacão entre as variáveis estimadas e observadas e o gráfico de erros de cada eauacão do modelo. A função Gama Generalizada apresentou ajustes satisfatórios aos dados. O sistema de equações para descrever a tendência dos parâmetros desta funcão também apresentou bom aiuste. Concluiu-se que a funcão Gama Generalizada gera estimativas consistentes e precisas e pode ser utilizada para a modelagem da distribuição diamétrica em povoamentos de eucalipto.

Palavras-chave: Modelagem florestal; função densidade de probabilidade; prognose.
\end{abstract}

\section{Abstract}

Generalized gamma distribution to describe diameter distribution of a stand of eucalyptus. This work focuses on the parameters estimation of generalized gamma distribution to describe the diameters distribution of a no thinning equiano eucalvptus stand and evaluate the biological realitv of a equations svstem to design such parameters and describe this distribution in a future age. Were used 48 plots with four measurements per year, from an experiment installed in a Eucalvptus grandis x Eucalvptus urophvlla hvbrid stand. located in the northeastern of Bahia state. The equations system to projection for the parameters was assessed using the correlation coefficients between the estimated and observed variables and the errors oraph for each eauation of model. The generalized gamma distribution presented satisfactorv adiustments to the data. The eauations system to describe the parameters tendency of this function also showed good adjustment. It was concluded that the generalized gamma function generates consistent and accurate estimates and can be used for modeling the diameter distribution in eucaly ptus stands.

Keywords: Forest modeling; probability density function; prognosis.

\section{INTRODUÇÃ̃O}

A distribuição diamétrica, em que há o agrupamento dos diâmetros das árvores (DAP) em classes, representa uma ferramenta simples e, ao mesmo tempo, eficiente para caracterizar a estrutura de uma floresta. Is so se deve ao fato de o diâmetro se correlacionar bem com outras variáveis importantes do povoamento, como, por exemplo, altura e volu me, entre outros (ARCE, 2004).

A dinâmica do crescimento e da produção por classe de diâmetro pode ser estimada por meio de modelos de distribuição diamétrica (MDD). Segundo Campos e Leite (2013), esses modelos estimam o nú mero de árvores por hectare, por classe de diâmetro nas idades presente e futura, permitindo estimar a produção por classe, subsidiando as decisões em que há interesse em quantificar multiprodutos da madeira.

A principal característica de um MDD é o emprego de função densidade de probabilidade (f.d.p.), pela qual se define a probabilidade de frequência associada com cada centro de classe diamétrica. Depois de estimados os parâmetros da f.d.p., eles são correlacionados às características do povoamento para, assim,

FLOREST A, Curitiba, PR, v. 46, n. 1, p. 67 - 73, jan. / mar. 2016.

Castro, R. V. O. etal.

ISSN eletrônico 1982-4688

DOI: $10.5380 /$ rf.v46il .40558 
prognosticar a distribuição des se povoamento em idades futuras (CAMPOS; LEITE, 2013), podendo todas essas características ser analis adas simu ltaneamente, ao longo do tempo.

Existem hoje na literatura florestal diversas f.d.p. utilizadas para modelagem da distribuição diamétrica em florestas naturais e plantadas. Podemos destacar as funções Log-Normal (DEB et al., 2014), Beta (CLUTTER; BENNETT, 1965), Gama (ARAÚJO JÚNIOR et al., 2013), Hiperbólica (BINOTI et al., 2011; BINOTI et al., 2014), Weibull (LEITE et al., 2006; MIGUEL et al., 2010; BINOTI et al., 2010), S Johnson (SILVA et al., 2009; TÉO et al., 2012), Normal e Exponencial (BAILEY, 1980).

Gama Generalizada é uma f.d.p. mais nova (1962) que a distribuição Gama (1836) e a distribuição Weibull (1939). Foi introduzida por Stacy e Mihran (1965), a fim de combinar a eficiência dessas duas distribuições (GOMES et al., 2008).

Segundo Gomes et al. (2008), a função Gama Generalizada de três parâmetros é expressa por Função Densidade de Probabilidade (1) e Função Acumulada da Distribuição (2).

$$
\begin{aligned}
f(x) & =\frac{k x^{k \alpha-1}}{\beta^{k \alpha} \Gamma(\alpha)} \exp \left(-(x / \beta)^{k}\right) \\
F(x) & =\frac{\Gamma_{(x / \beta)^{k}}(\alpha)}{\Gamma(\alpha)}
\end{aligned}
$$

em que: $f(x)=$ frequência na classe $x ; F(x)=$ frequência acumulada na classe $x ; k$ e $\alpha=$ parâmetros de forma, $(k>0 ; \alpha>0) ; \beta=$ parâmetro de escala, $\beta>0 ; \Gamma=$ função Gama.

Alguns exemp los para demonstrar sua flexibilidade foram ilustrados na figura 1. A distribuição mostrase sensível para captar diferentes distribuições.
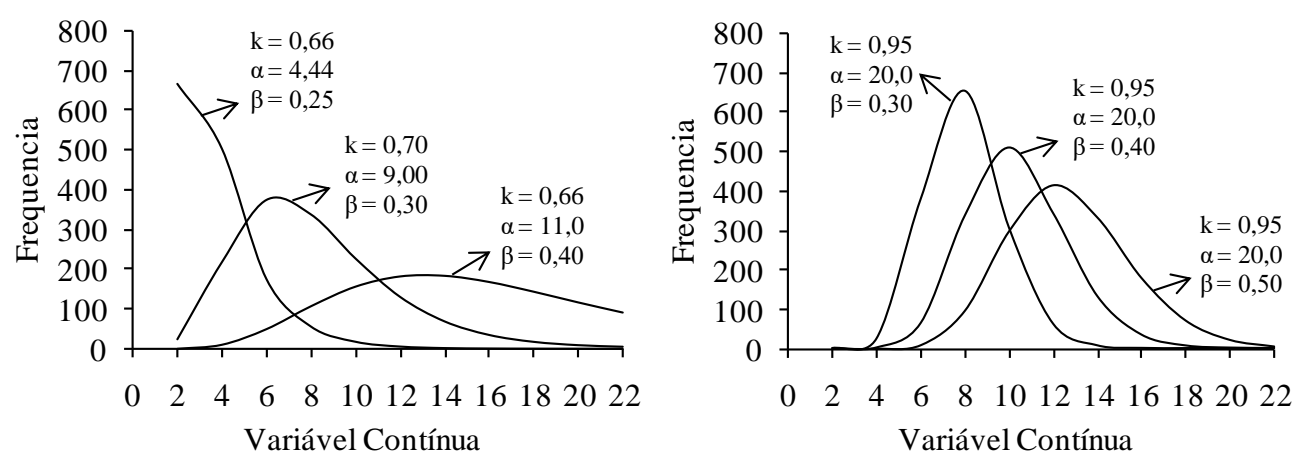

Figura 1. Sensibilidade dos parâmetros da função Gama Generalizada para descrever a distribuição de uma variável contínua.

Figure 1. Sensitivity of parameters of the Generalized Gamma function to describe the distribution of a continuous variable.

Trata-se de uma distribuição popular em diversas áreas, tais como engenharia civil (PHAN; ALMHANA, 1995) e economia (KLEIBER; KOTZ, 2003), por ser extremamente flexível, mas não foi encontrada na literatura para descrever a distribuição diamétrica de povoamentos florestais. Assim, o objetivo do presente estudo foi estimar parâmetros da função Gama Generalizada para descrever a distribuição de diâmetros de um povoamento equiâneo de eucalipto não desbastado e avaliar a realidade biológica de um sistema de equações para projetar tais parâmetros e descrever essa distribuição emu ma idade futura.

\section{MATERIAL E MÉTODOS}

Os dados utilizados foram provenientes de um povoamento do híbrido Eucalyptus urophylla $\mathrm{x}$ Eucalyptus grandis, localizado na região nordeste do estado da Bahia. Eles são referentes às medições de 48 parcelas permanentes nas idades de 27, 40, 50 e 58 meses, com área útil de aproximadamente $2.600 \mathrm{~m}^{2}$ por unidade amostral. Em cada medição foram registrados os diâmetros a 1,3 m do solo (DAP) de todas as árvores contidas nas parcelas.

Os parâmetros foram estimados por máxima verossimilhança, utilizando-se o software EasyFit, e as distribuições diamétricas foram estimadas pela função Gama Generalizada para cada parcela em cada idade, sendo testadas a consistência do ajus te pelo tes te não paramétrico de Kolmogorov-Smirnov (KS) a 5\% de probabilidade (3): 


$$
D=\operatorname{máx}\left|F_{o}(x)-F_{e}(x)\right|
$$

em que: $\mathrm{D}=$ diferença máxima absoluta; $\mathrm{F}_{\mathrm{o}}(\mathrm{x})$ e $\mathrm{F}_{\mathrm{e}}(\mathrm{x})$ = freqüências acumuladas observadas e esperadas, respectivamente.

Em seguida foi calculada uma matriz de correlação entre os parâmetros da função e atributos do povoamento e idade.

A partir das distribuições diamétricas observadas na idade de 27 meses, foram recuperadas as distribuições para as idades de 40,50 e 58 meses, por meio do modelo de distribuição diamétrica ajustado, a fim de serem comparadas, também pelo teste $\mathrm{KS}$, as distribuições recuperadas com as observadas em cada amplitude, conforme Nogueira et al. (2005), Leite et al. (2006) e Binoti et al. (2010). A seguir, é apresentado o sistema de equações (4-8) selecionado para a modelage m:

$$
\begin{gathered}
\alpha_{2}=\phi_{3}+\alpha_{1}\left(I_{1} / I_{2}\right) \phi_{4}+\phi_{5}\left(q_{2}\right)+\varepsilon \\
\operatorname{Beta}_{2}=\phi_{6}+\operatorname{Beta}_{1}\left(I_{1} / I_{2}\right) \phi_{7}+\phi_{8}\left(q_{2}\right)+\varepsilon \\
K_{2}=\phi_{0}+K_{1}\left(I_{1} / I_{2}\right) \phi_{1}+\phi_{2}\left(q_{2}\right)+\varepsilon \\
N_{2}=N_{1} \cdot e^{-\phi_{12}\left(I_{2}^{\phi_{13}}-I_{2}^{\phi_{13}}\right)}+\varepsilon \\
q_{2}=\phi_{9}+q_{1}\left(I_{1} / I_{2}\right) \phi_{10}+\phi_{11}\left(N_{2}\right)+\varepsilon
\end{gathered}
$$

em que: $I_{1}$ e $I_{2}=$ idades, inicial e final, respectivamente; $\mathrm{q}_{1}$ e $\mathrm{q}_{2}=$ diâmetros médios observados por parcela nas idades inicial e final; $N_{1}$ e $N_{2}=$ freqüências totais observadas por parcela nas idades inicial e final; $K$, $\alpha$ e $\beta=$ parâmetros da função; $\phi_{1}$ a $\phi_{13}=$ parâmetros a serem estimados; $\varepsilon=$ erro aleatório; as dema is, conforme definidas anteriormente.

\section{RES ULTADOS E DISCUSSÃO}

Na figura 2 é apresentada a tendência dos parâmetros da função Gama Generalizada em função da idade, obtidos após o ajuste. Todos os ajustes foram precisos, avaliados pelo teste $\mathrm{KS}(\mathrm{p}<0,05)$. Assim, a função Gama Generalizada mostrou-se flexível o suficiente para descrever as tendências de todas as parcelas, em todas as idades.

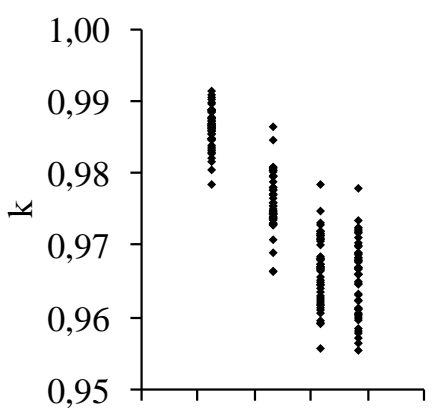

$12 \quad 24 \quad 36 \quad 48 \quad 60 \quad 72$

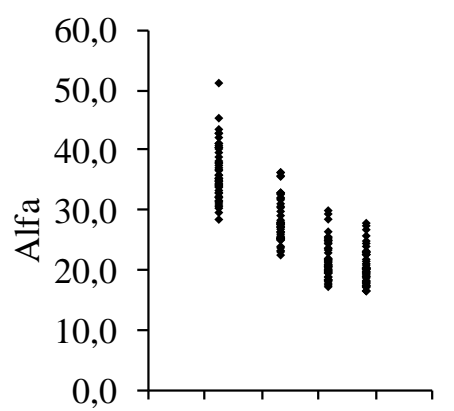

$\begin{array}{lllllll}12 & 24 & 36 & 48 & 60 & 72\end{array}$

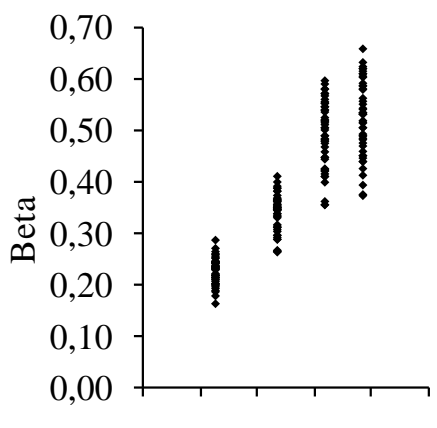

$12 \quad 24 \quad 36 \quad 48 \quad 6072$

Idade (meses)

Idade (meses)

Figura 2. Tendência dos parâmetros da função Gama Generalizada ajustada para as 48 parcelas nas quatro med ições sucessivas.

Figure 2. Tendency of parameters Generalized Gamma function fitted for the 48 plots in the four successive measurements.

Pode-se esperar que todos os parâmetros que compõem a função Gama Generalizada sejam correlacionados com a idade do povoamento, haja vista a tendência bem definida apresentada na figura 2. Para verificação estatística, na tabela 1 é apresentada a matriz de correlação entre os parâmetros da função e a idade, bem co mo entre os parâmetros e as características do povoamento.

FLOREST A, Curitiba, PR, v. 46, n. 1, p. 67 - 73, jan. / mar. 2016

Castro, R. V. O. etal.

ISSN eletrônico 1982-4688

DOI: $10.5380 /$ rf.v46il .40558 
Tabela 1. Matriz de correlação entre os parâmetros da função Gama Generalizada e as variáveis idade e caracterís ticas do povoamento.

Table 1. Correlation matrix between the Generalized Gamma function parameters and variables age and stand characteristics.

\begin{tabular}{lcccccc}
\hline Parâmetro & Idade & Dmín & Dmáx & $\mathrm{q}$ & $\mathrm{AB}$ & \\
\hline $\mathrm{k}$ & $-0,88^{*}$ & $-0,02$ & $-0,91^{*}$ & $-0,93^{*}$ & $-0,90^{*}$ & $0,16^{*}$ \\
Alfa & $-0,85^{*}$ & 0,01 & $-0,87^{*}$ & $-0,82^{*}$ & $-0,85^{*}$ & $-0,08$ \\
Beta & $0,90^{*}$ & 0,04 & $0,93^{*}$ & $0,89^{*}$ & $0,91^{*}$ \\
\hline
\end{tabular}

*Correlações significativas, a $5 \%$ de probabilidade, peloteste t.

Dmín = diâmetro mínimo da parcela $(\mathrm{cm}) ;$ Dmáx = diâmetro máximo da parcela $(\mathrm{cm}) ; \mathrm{q}=$ diâmetro médio da parcela $(\mathrm{cm}) ;$ $\mathrm{AB}=$ área basal da parcela $\left(\mathrm{m}^{2} / \mathrm{ha}\right) ; \mathrm{N}=$ número de árvores/ha; demais variáveis conforme descritas anteriormente.

Os parâmetros foram altamente corre lacionados com as variáveis, em especial a idade, Dmáx, q e AB, o que indica que as variáveis que compõem o sistema de equações selecionado podem ser utilizadas para projeção desses parâmetros para idades futuras, pois têm influência sobre as variáveis dependentes, bem co mo observado por Nogueira et al. (2005), utilizando a função Weibull para estimar a distribuição diamétrica em povoamentos de Eucalyptus submetidos a desbaste, e por Araújo Júnior et al. (2013), utilizando a função Gama para estimar a distribuição diamétrica em povoamentos de Eucalyptus. O ajuste do sistema é o que segue (Tabela 2).

Tabela 2. Sistema de equações ajustado e seus respectivos coeficientes de determinação $\left(\mathrm{R}^{2} \%\right)$.

Table 2. System of equations fitted and their coefficients of determination $\left(\mathrm{R}^{2} \%\right)$.

\begin{tabular}{ccc}
\hline Modelo n $^{\mathbf{0}}$ & \multicolumn{1}{c}{ Equação } & $\mathbf{R}^{\mathbf{2} \%}$ \\
\hline (4) & $\alpha_{2}=22,7989+\alpha_{1}\left(I_{1} / I_{2}\right) 0,9221-1,6517\left(q_{2}\right)$ & 91,89 \\
(5) & Beta $_{2}=-0,1118+$ Beta $_{1}\left(I_{1} / I_{2}\right) 0,5554+0,0343\left(q_{2}\right)$ & 90,41 \\
(6) & $K_{2}=1,0458+K_{1}\left(I_{1} / I_{2}\right) 0,0068-0,0069\left(q_{2}\right)$ & 82,46 \\
(7) & $N_{2}=N_{1} \cdot e^{+0.1034\left(I_{2}^{0.0153}-I_{2}^{0.0153}\right)}$ & 96,77 \\
(8) & $q_{2}=12,4796+q_{1}\left(I_{1} / I_{2}\right) 0,3782-0,0113\left(N_{2}\right)$ & 89,62 \\
\hline
\end{tabular}

A dispersão dos resíduos referentes ao ajuste é apresentada na figura 3.

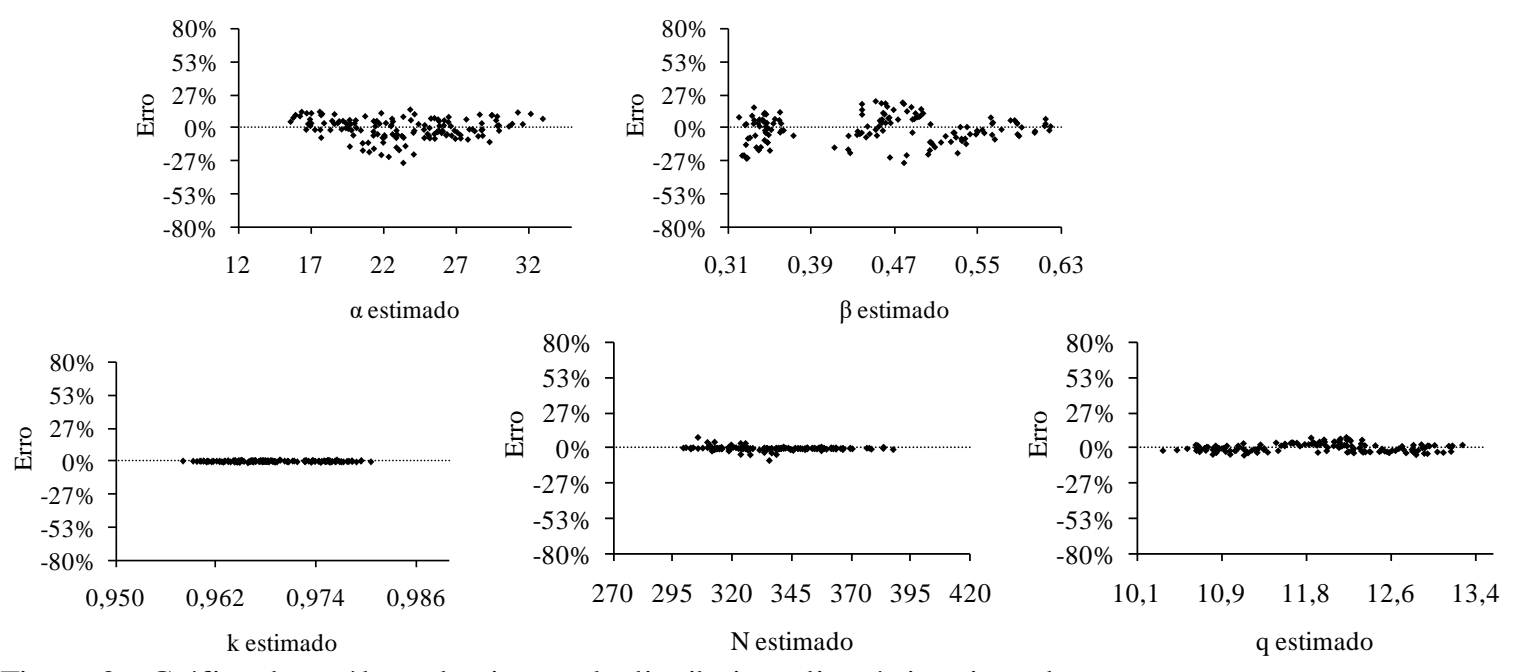

Figura 3. Gráfico de resíduos do sistema de distribuição diamétrica ajustado.

Figure 3. Graphical evaluation of residues of the diameter distribution system fitted. 
Os resíduos apresentados na figura 3 mostraram que as estimativas dos parâmetros $\alpha, \beta, \mathrm{k}$ não foram tendenciosas. Para validar a eficiência da projeção, aplicou-se o sistema de equações para a função Gama Generalizada nos dados da primeira medição das parcelas (27 meses) e foram recuperados os parâmetros para as idades posteriores (40, 50 e 58 meses).

Com relação à prognose da distribuição diamétrica, obtida pela recuperação dos parâmetros de cada parcela nas idades futuras, o teste de KS mostrou-se não significativo ( $p<0,05$ ) em todas as idades, para 95,8\% das projeções, o que permitiu aceitar a hipótese de que existe concordância entre as distribuições prognosticadas e observadas. Foram selecionadas duas parcelas (3 e 41) para ilustração da projeção da distribuição diamétrica (Figura 4).

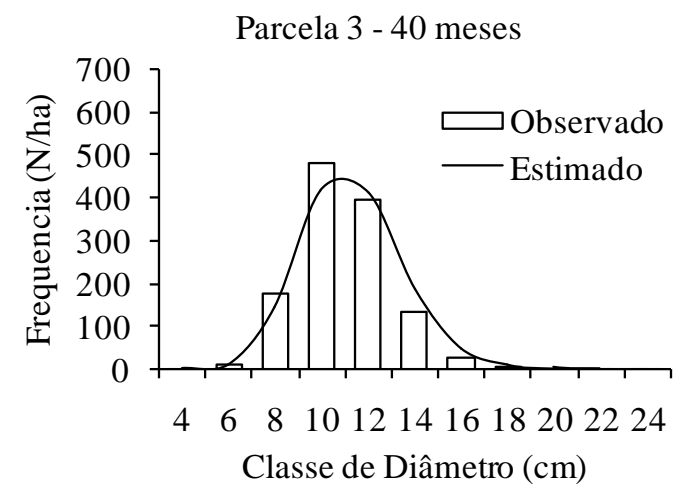

Parcela 3 - 50 meses

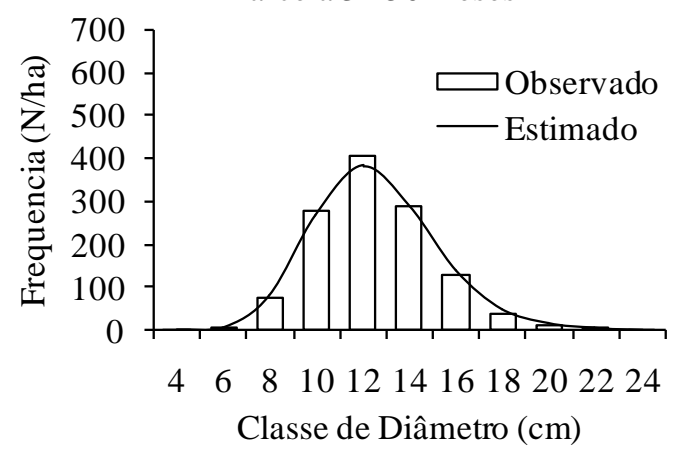

Parcela 3 - 58 meses

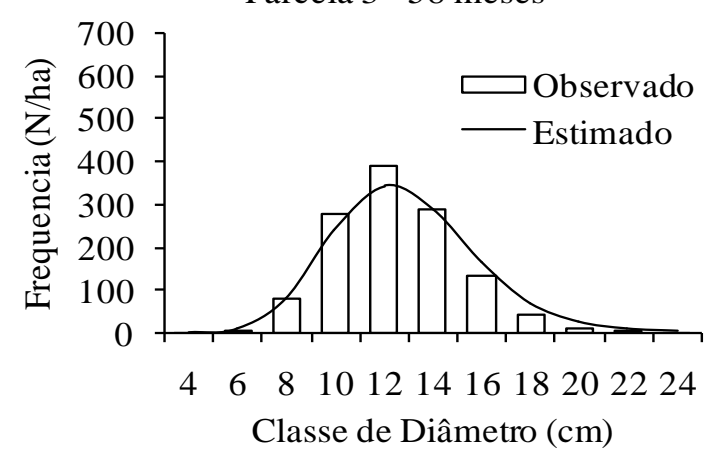

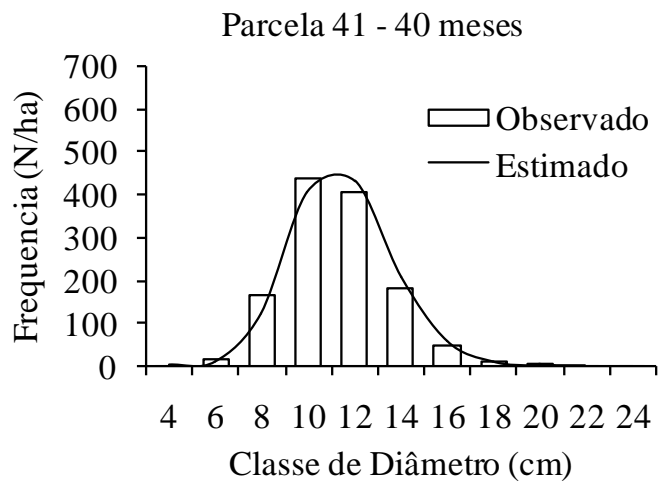

Parcela 41 - 50 meses

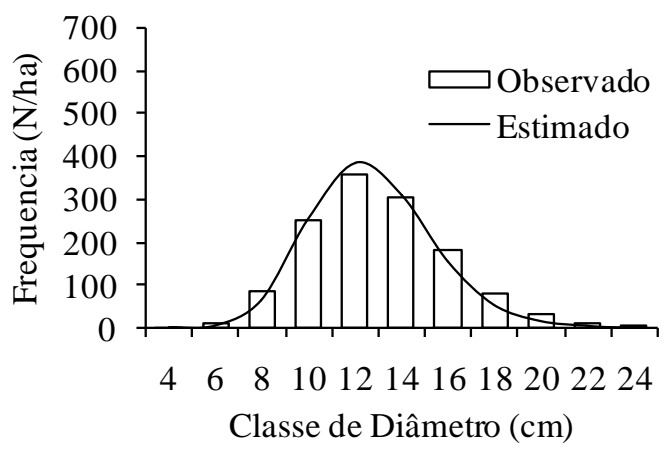

Parcela 41 - 58 meses

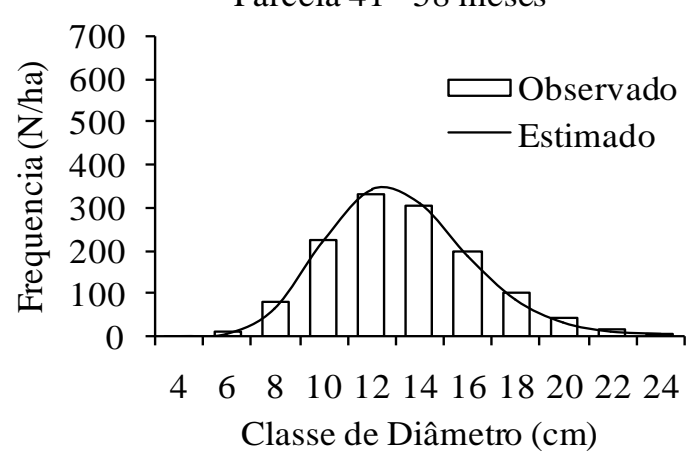

Figura 4. Recuperação da distribuição diamétrica referente às parcelas 3 e 41, a partir da medição inicial (27 meses) para as idades de 40, 50 e 58 meses, utilizando o MDD ajustado.

Figure 4. Recovery of the diameter distribution referring to the plots 3 and 41 , from the initial measurement (27 months) for ages 40, 50 and 58 months, using the diameter distribution model fitted.

As distribuições de diâmetros obtidas seguiram o comporta mento natural da distribuição diamétrica para povoamentos equiâneos, ou seja, em idades maiores ocorre o achatamento das curvas, com o aumento do número de árvores nas maiores classes de diâmetros, ou seja, há um deslocamento da curva para a direita

FLOREST A, Curitiba, PR, v. 46, n. 1, p. 67 - 73, jan. / mar. 2016.

Castro, R. V. O. etal.

ISSN eletrônico 1982-4688

DOI: $10.5380 / \mathrm{rf} . v 46 \mathrm{il} .40558$ 
(LEITE et al., 2005; NOGUEIRA et al., 2006). As áreas correspondentes aos ingressos de árvores em sucessivas classes de diâmetro diminuíram com o passar do tempo, em função do aumento da competitividade entre as árvores. Na figura 5 foram compiladas todas as distribuições projetadas para a Parcela 3, para facilitar essa compreensão.

Assim, pôde-se inferir que o modelo de distribuição diamétrica foi adequado e coerente, do ponto de vista tanto biológico quanto estatístico, para a projeção da distribuição diamétrica do povoamento de eucalipto avaliado, corroborando assim a informação de Ramos (2014) de que a distribuição Gama Generalizada se mostra umótimo modelo probabilístico que leva a excelentes inferências e ajustes em dados reais.

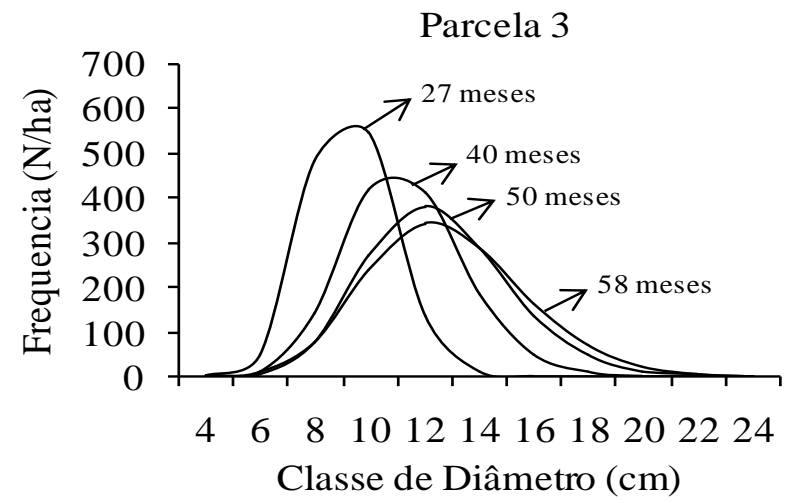

Figura 5. Recuperação da distribuição diamétrica referente à Parcela 3, a partir da medição inicial (27 meses) para as idades de 40, 50 e 58 meses, utilizando o MDD ajustado.

Figure 5. Recovery of the diameter distribution referring to the plot 3 , from the initial measure ment ( 27 months) for ages 40, 50 and 58 months, using the diameter distribution model fitted.

\section{CONCLUS ÕES}

- A função densidade de probabilidade Gama Generalizada é adequada para descrever a distribuição diamétrica de povoamentos equiâneos de eucalipto em dife rentes idades.

- O modelo de distribuição diamétrica foi adequado e coerente, do pon to de vista biológico, para a projeção da dis tribuição dia métrica do povoamento de eucalipto avaliado.

\section{AGRADECIMENTOS}

Os autores agradecem à Fundação de Amparo à Pesquisa do Estado de Minas Gerais (FAPEMIG) e ao Conselho Nacional de Desenvolvimento Científico e Tecnológico (CNPq), pelo apoio financeiro.

\section{REFERÊNCIAS}

ARAÚJO JÚNIOR, C. A.; LEITE, H. G.; CASTrO, R. V. O.; BINOTI, D. H. B.; AlCÂNTARA, A. E. M.; BINOTI, M. L. M. S. Modelagem da distribuição diamétrica de povoamentos de eucalipto utilizando a função Gama. Cerne, Lavras, v. 19, n. 2, p. 307 - 314, 2013.

ARCE, J. E. Modelagem da estrutura de florestas clonais de Populus deltoides Marsh. através de distribuições diamétricas probabilísticas. Ciência Florestal, v. 14, n. 1, p.149 - 164, 2004.

BAILEY, R. L. Individual tree growth derived from diameter distribution models. Forest Science, Amsterdam, v. 26, n. 4, p. $626-632,1980$.

BINOTI, D. H. B.; BINOTI, M. L. M. S.; LEITE, H. G. Avaliação da função hiperbólica truncada para a descrição da distribuição diamétrica de povoamentos desbastados de eucalipto. Revista Árvore, Viçosa-MG, v. 38, n. 6, p. 1037 - 1044, 2014.

BINOTI, D. H. B.; LEITE, H. G.; GUIMARÃES, D. P.; SILVA, M. L. M.; GARCIA, S. L. R.; FA RDIN, L. P. Eficiência das funções Weibull e Hiperbólica para descrição de distribuições diamétricas de povoamentos de Tectona grandis. Revista Árvore, Viçosa/MG, v. 35, n. 2, p. 299 - 306, 2011.

BINOTI, D. H. B.; LEITE, H. G.; NOGUEIRA, G. S.; SILVA, M. L. M.; GARGIA, S. L. R.; CRUZ, J. P. Uso 
da função Weibull de três parâmetros em um modelo de distribuição diamétrica para plantios de eucalipto submetidos a desbaste. Revista Árvore, Viçosa/MG, v. 34, n. 1, p. 147 - 156, 2010.

CAMPOS, J. C. C.; LEITE, H. G. Mensuração florestal : perguntas e respostas. 4. ed. UFV, 2013, 605 p.

CLUTTER, J. L.; BENNETT, F. A. Diameter distributions in old-field slash pine plantations. Georgia Forest Research Council Report, n .13, p. 1 - 9, 1965.

DEB, J. C.; SALMAN, H. R.; ABDUL, H.; CHOWDHURY, Q.; ROY, A. Characterising the diameter distribution of Sal plantations by comparing normal, lognormal and Weibull distributions at Tilagarh Eco-park, Bangladesh. Southern Forests, v. 76, n. 4, p. 201 - 208, 2014.

EasyFit - Mathwave data analsys \& simulation. 〈http://www.mathwave.co m/products/eas yfit.html >. Acesso em: 05 mar. 2015

GOMES, O.; COMBES, C.; DUSSAUCHOY, A. Parameter estimation of the generalized gamma distribution. Mathematics and Computers in Simul ation, v. 79, n. 4, p. 955 - 963, 2008.

KLEIBER, S.; KOTZ, S. Statistical Size Distributions in Economics and Actuarial Sciences. Wiley-Interscience, 2003, ISBN 0-471 - 15064-9.

LEITE, H. G.; NOGUEIRA, G. S.; CAMPOS, J. C. C.; SOUZA, A. L.; CARVALHO, A. Avaliação de um modelo de distribuição diamétrica ajustado para povoamentos de Eucalyptus sp. submetidos a desbaste. Revista Árvore, Viçosa, v. 29, n. 2, p. 271 - 280, 2005.

LEITE, H. G.; NOGUEIRA, G. S.; CAMPOS, J. C. C.; TAKIZAWA, F. H.; RODRIGUES, F. L. Um modelo de distribuição diamétrica para povoamentos de Tectona grandis submetidos a desbaste. Revista Árvore, Viçosa/MG, v. 30, n. 1, p. 89 - 98, 2006.

MIGUEL, E. P.; MACHADO, S. A.; FIGUEIREDO FILHO, A.; ARCE, J. E. Using the Weibull function for prognosis of yield by diameter class in Eucalyptus urophylla stands. Cerne, Lavras, v. 16, n. 1, p. 94 - 104, 2010.

NOGUEIRA, G. S.; LeITE, H. G.; CAMPOS, J. C. C.; CARVALhO, A. F.; SOUZA, A. L. Modelo de distribuição diamétrica para povoamentos de Eucalyptus sp. submetidos a desbaste. Revista Árvore, Viçosa/MG, v. 29, n. 4, p. 579 - 589, 2005.

PHAN, T.; ALMHANA, J. The generalized gamma distribution: its hazard rate and stress -strength model. IEEE Transactions on Reliability 44, 1995.

RAMOS, P. L. Aspectos computacionais para inferência na distribuição gama generalizada. 165 f. Dissertação (Mestrado em Matemática Aplicada e Computacional) - Universidade Estadual Paulista "Júlio de Mesquita Filho", Presidente Prudente, 2014.

SILVA, V. S. M.; SOARES, T. S.; COLPINI, C.; TRA VA GIN, D. P.; HOSOKAWA, R. T.; SCOLFORO, J. R. S. Prognose da produção de Eucalyptus camaldulensis Dehnh. pela aplicação da função de distribuição $S_{B}$ de Johnson. Re vista Árvore, v. 33, n. 5, p. 853 - 863, Viçosa, MG, 2009.

STACY, E. W.; MIHRAN, G. A. Parameter estimation for a generalized gamma distributions. Technometrics, v. 7, n. 3, p. $349-358,1965$

TÉO, S. J.; BIANCHI, J. C.; PELOSO, A.; NAVA, P. R.; MARCON, A.; EHLERS, T.; COSTA, R. H. Desempenho de funções de densidade probabilísticas para descrever a distribuição diamétrica de Pinus taeda, na região de Caçador, SC. Floresta, Curitiba, v. 42, n. 4, p. 741 - 754, 2012. 